\title{
Deployment Optimization of Connected and Automated Vehicle Lanes with the Safety Benefits on Roadway Networks
}

\author{
Zhibo Gao, ${ }^{1}$ Zhizhou Wu $\mathbb{D}^{1}{ }^{1}$ Wei Hao $\mathbb{D}^{2,3}$ and Kejun Long ${ }^{2,3}$ \\ ${ }^{1}$ The Key Laboratory of Road and Traffic Engineering, Ministry of Education, Tongji University, 4800 Cao'an Road, \\ Shanghai 201804, China \\ ${ }^{2}$ School of Traffic \& Transportation Engineering, Changsha University of Science \&Technology, Changsha 410076, China \\ ${ }^{3}$ Hunan Key Laboratory of Smart Roadway and Cooperative Vehicle-Infrastructure Systems, \\ Changsha University of Science \& Technology, Changsha 410205, China
}

Correspondence should be addressed to Zhizhou Wu; wuzhizhou@tongji.edu.cn and Wei Hao; haowei@csust.edu.cn

Received 3 January 2020; Revised 17 March 2020; Accepted 10 July 2020; Published 1 August 2020

Academic Editor: Jaeyoung Lee

Copyright (c) 2020 Zhibo Gao et al. This is an open access article distributed under the Creative Commons Attribution License, which permits unrestricted use, distribution, and reproduction in any medium, provided the original work is properly cited.

Reasonable deployment of connected and automated vehicle (CAV) lanes which separating the heterogeneous traffic flow consisting of both CAVs and human-driven vehicles (HVs) can not only improve traffic safety but also greatly improve the overall roadway efficiency. This paper simplified CAV lane deployment plan into the problem of traffic network design and proposed a comprehensive decision-making method for CAV lane deployment plan. Based on the traffic equilibrium theory, this method aims to reduce the travel cost of the traffic network and the management cost of CAV lanes using a bilevel primary-secondary programming model. In addition, the upper level is the decision-making scheme of the lane deployment, while the lower level is the traffic assignment model including CAV and HV modes based on the decision-making scheme of the upper level. After that, a genetic algorithm was designed to solve the model. Finally, a medium-scaled traffic network was selected to verify the effectiveness of the proposed model and algorithm. The case study shows that the proposed method obtained a feasible scheme for lane deployment considering from both the system travel cost and management cost of CAV lanes. In addition, a sensitivity analysis of the market penetration rate of CAVs, traffic demand, and the capacity of CAVLs further proves the applicability of this model, which can achieve better allocation of system resources and also improve the traffic efficiency.

\section{Introduction}

Recently, connected and automated driving technology has attracted the attention of automobile enterprises, universities, and scientific research institutions due to the great function of intelligent networking technology in improving traffic safety $[1,2]$, road capacity $[3,4]$, energy consumption [5-7], driving experience [8], etc. In addition, the development of connected and automated driving technology has prompted the rapid progress of a new generation of intelligent transportation systems [9]. It can be predicted for a considerable time to come with the scenario for a traffic development mode coexisting with CAVs and HVs.

As a new generation of the automobile, CAVs have natural differences in driving behavior compared with HVs.
They need more accurate environmental perception, less headway, and shorter reaction times with following and changing lanes [10]. Currently, the main control strategies for CAV technology focus on adaptive cruise control (ACC) and cooperative adaptive cruise control (CACC). First of all, ACC strategy is to obtain the acceleration and speed of the front vehicle through on-board detection equipment (via vehicle-to-vehicle $(\mathrm{V} 2 \mathrm{~V})$ communication) and realize acceleration optimization control through the ACC control system. Secondly, CACC is based on ACC and realizes vehicle formation via $\mathrm{V} 2 \mathrm{~V}$ technology to maintain a smaller headway, thus can greatly improving traffic efficiency [11-13]. Due to truck drivers experience significantly higher risk of suffering serious injury and fatality than passenger vehicle drivers [14, 15], a number of machine learning 
models were established to examine crash severity on roadway segments $[16,17]$. Until now, truck CACC is believed as one of the potentially effective solutions to these challenges. However, CACC will be affected by HVs or other emergencies, when the CACC market penetration rate (MPR) is low. In addition, the formation of CACC vehicles will deactivate and switch to ACC or human-driven mode, which results in a drop for the road's vehicle capacity. Zeng et al. [18] studied the impairment of capacity caused by the failure of a CACC formation in the freeway merging area. The simulation results showed that the reduction of capacity in the merging area is $15.4 \%-17.2 \%$ under the same MPR compared with the pipeline capacity. In addition, Qin et al. [19] established the fundamental diagram model for the heterogeneous traffic flow of CACC vehicles mixed with ACC vehicles and found that the capacity of the heterogeneous traffic flow is lower than an HV traffic flow situation when the CACC MPR is less than $40 \%$.

In order to better accommodate CAVs, some scholars considered providing special roadway rights for CAVs so that they make a separation of CAVs from the mixed traffic, such as the Connected and Automated Vehicle Lanes (CAVLs) which are studied in this paper. In this strategy, CAVs are supposed to use the dedicated lane on which homogeneous traffic flow of CAVs is created [20]. On the contrary, setting CAVL will reduce the number of lanes for accommodating other HVs. If the CAVL deployment is not set properly, it will lead to a great waste of road system resource and cause drastic congestion in the traffic flow and decreases the overall performance of the road. For system planning, policymakers are interested in understanding possible sets of system enhancement options to meet their performance goals and obtain the most cost-effective deployment strategies for the future. Therefore, to improve the capacity and safety characteristics of existing traffic facilities, how to design and deploy CAVLs become an urgent problem for policymakers.

In traffic flow research, one important problem has aroused much attention: how the impact in roadway capacity will evolve as the connected and automated driving technologies mature and the penetration rate gradually increases? Some existing studies provide effective methods to solve this issue. During the theoretical research, Ghiasi et al. [21] developed an analytical capacity model to calculate the impact of different CAV technology scenarios and determine the optimal number of dedicated CAV lanes using the Markov chain method. Chen et al. [22] presented a mathematical framework to optimize a time-dependent deployment plan of autonomous vehicle lanes on a transportation network with heterogeneous traffic stream. In their work, the per-lane capacity can become tripled when it is converted from a regular lane to an AV lane. In order to shed light on how traffic operational capacity will change with the introduction of AVs, Chen et al. [23] developed a general theoretical framework to determine the valid domains of different lane policies and, more generally, AV distributions across lanes with respect to demand, as well as optimal solutions to accommodate AVs. Simulation is another significant method which can be utilized to investigate this problem. Liu et al. [24] analyzed the influence of the CAVL strategy on multilane freeway facilities under the mixed traffic flow. The analysis results showed that the strategy of CAVs lanes can improve pipeline capacity by $22 \%$ compared with conventional strategy with the CACC MPR approaching 60\%. Talebpour et al. [25] examined the impacts of reserving one lane of a four-lane highway for AVs on traffic flow dynamics and travel time reliability. It was found that throughput can be improved significantly if the AV penetration rate is greater than $30 \%$. Ye and Yamamoto [20] investigated the performance of traffic flow under different numbers of CAV dedicated lanes, compared it with mixed flow situation, and found that the benefit of setting CAVLs can only be obtained within a medium density range.

However, it is well known from "Braess" paradox $[26,27]$ that unilaterally improving the capacity of an existing link or adding a new link in the network instead of reducing the unit travel cost within the network. Therefore, some scholars turned to study the CAVL deployment problem for the transport network level. For example, Chen et al. [28] developed a mathematical framework to optimally design AV zones and developed a mixed-integer bilevel programming model to optimize the deployment plan. However, there is limited systematic research talking about the optimization of CAVLs deployment considering the total travel expense and the management cost of CAVLs.

As a result, this paper proposes an optimization method for CAVL deployment plan considering from the viewpoints of the whole traffic network. The objective of this method is to reduce the travel cost of the traffic network as well as the management cost of CAVLs. A primary-secondary method with a bilevel programming model is established. The upper level is the decision-making scheme of lane deployment, while the lower level assigns traffic flow including CAV mode and HV mode using the upper-level scheme. Based on the characteristics of the model, a genetic algorithm is creatively designed to solve the abovementioned models, and a medium-sized network is listed as an example to be analyzed in this research.

The remainder of this paper is structured as follows. Section 2 presents the mathematical formulation to optimize the CAVLs deployment plan and to describe the flow distributions of both CAVs and HVs. Section 3 designs a genetic algorithm to solve the proposed bilevel programming optimization model. Section 4 conducts numerical studies and sensitivity analysis. Finally, conclusions and recommendations are delivered in Section 5.

\section{Mathematical Formulation}

2.1. Problem Description. "Connected and automated vehicle lanes" refer to a lane management method that provides the exclusive right for CAVs to travel on some links of the traffic network according to the traffic demand. The following conditions need to be considered when setting CAVLs: the first condition is lane conditions, which exists at least two or more lanes going on the same direction and there is no interference from other traffic modes besides HVs; the second condition is the traffic conditions, which 
the basic capacity of HV lanes will not be affected by the CAVLs; and the third one is the link conditions, which the link has the construction conditions needed for CAVLs, such as the requirements for the layout of communication equipment and the construction cost.

One of the key points for CAVL deployment is that the decision-making scheme for every kind of lane should be coordinated in order to achieve the best combination effect. The best combination effect will be achieved only after setting a reasonable scale on the network. The second key point is that travellers will choose the most advantageous route for themselves according to the well-established CAVL schemes, and the traffic flow can be balanced under the current network conditions. The third consideration is that the traffic organizer will optimize the scheme of the CAVLs based on the equilibrium state caused by the travellers route choice behavior. Finally, a master-slave game (also known as a Stackelberg game) is formed between the traffic organizers and the travellers.

In summary, CAVL deployment is a systematic problem that is really necessary to consider the deployment, considering from the level of the traffic network. In addition, the master-slave game relationship exists between traffic planning organizers and travellers. Finally, a comprehensive decision will be achieved based on the traffic equilibrium theory.

2.2. Assumptions and Definitions. The analyzed model established in this section is based on the following assumptions:

(i) There are only two kinds of managed lanes in this research: CAVLs and HV lanes

(ii) The topology of the traffic network is predefined known

(iii) The traffic demand among the origin-destination (OD) pairs is predefined known and unsaturated

(iv) The scope of CAVs cooperation could not affected by V2V communication distance in CAVLs, that is, CAVs are all fully communicated in each CAVLs

\subsection{Primary-Secondary Method with Bilevel Programming} Model. For the optimization problem of CAVL deployment, the traveller aims to minimize the travel cost or travel time, while the government planning department enhances how to design or improve the traffic network under a limited investment to maximize the performance of a certain system. With the need of comprehensive decision-making for two different lanes, this paper establishes a primary-secondary bilevel programming model. The upper level establishes the decision-making scheme for lane deployment, and the objective function is the system cost including the travel cost and the management cost of CAVLs. In addition, the lower level utilizes the User-Equilibrium (UE) model to describe the traffic flow assignment of the CAV mode and HV mode, respectively, according to the decision-making scheme of the upper level. The assignment results obtained from the route choice of two travel modes are used to evaluate the performance of the decision-making scheme.

Considering the constraints of the number of lanes and link capacity, a decision-making scheme to minimize the system cost is established in the upper-level planning. The formulation of the model is listed as follows:

$$
\begin{aligned}
& \min F\left(n_{a}^{\mathrm{Cv}}\right)= \sum_{a \in D}\left(x_{a}^{\mathrm{Cv}} t_{a}^{\mathrm{Cv}}+x_{a}^{\mathrm{Hv}} t_{a}^{\mathrm{Hv}}\right)+\sum_{a \in \bar{D}}\left[\left(x_{a}^{\mathrm{Cv}}+x_{a}^{\mathrm{Hv}}\right) t_{a}^{\mathrm{Hv}}\right] \\
&+\sum_{a \in A} w_{C} n_{a}^{\mathrm{Cv}} \\
& 1 \leq n_{a}^{\mathrm{Hv}} \leq n_{a}, \quad \forall a \in A \\
& 0 \leq n_{a}^{\mathrm{Cv}} \leq n_{a}-1, \quad \forall a \in A \\
& n_{a}=n_{a}^{\mathrm{Cv}}+n_{a}^{\mathrm{Hv}}, \quad \forall a \in A \\
& x_{a}= x_{a}^{\mathrm{Cv}}+x_{a}^{\mathrm{Hv}}, \quad \forall a \in A
\end{aligned}
$$

where equation (1) is the objective function; equations (2) and (3) are the constraints of the number of CAVLs and HV lanes, which ensure that the number of $\mathrm{HV}$ lanes is not less than one; equation (4) is the conservation of lane number; and equation (5) is the conservation of link flow. In equation (1), the first term is the sum travel cost of links with CAVLs, the second term is the sum travel cost of links without CAVLs, and the third term is the sum management cost of CAVLs.

In the lower-level planning, the flows should be differentiated in the route selection for the HV mode and CAV mode. In order to promote the CAV mode, CAV traffic flow is first loaded, initial cost of the unmodified links is updated when the network is balanced, and then HV traffic flow is loaded. Therefore, there is a primary-secondary relationship for the two traffic flow assignment models.

The assignment model of CAV traffic flow is listed as follows:

$$
\begin{gathered}
\min \quad \sum_{a \in A} \int_{0}^{x_{a}^{\mathrm{Cv}}} t_{a}^{\mathrm{Cv}}\left(x_{a}^{\mathrm{Cv}}, n_{a}^{\mathrm{Cv}}\right) \mathrm{d} \omega \\
\text { s.t. } \quad \sum_{k \in P_{\mathrm{rs}}} f_{\mathrm{rs}}^{\mathrm{Cv}, k}=q_{\mathrm{rs}}^{\mathrm{Cv}},(r, s) \in \mathrm{RS}, \\
f_{\mathrm{rs}, k}^{\mathrm{Cv}} \geq 0, \quad k \in P_{\mathrm{rs}},(r, s) \in \mathrm{RS}, \\
x_{a}^{\mathrm{Cv}}=\sum_{(r, s) \in \mathrm{RS}} \sum_{k \in P_{\mathrm{rs}}} f_{\mathrm{rs}}^{\mathrm{Cv}, k} \delta_{\mathrm{rs}}^{\mathrm{Cv}, k}, \quad a \in A \\
t_{a}^{\mathrm{Cv}}=t_{a}^{0}\left\{1+\alpha\left[\frac{x_{a}^{\mathrm{Cv}}}{n_{a}^{\mathrm{Cv}} C_{l}^{\mathrm{Cv}}+\left(1-\eta_{a}\right) n_{a} C_{l}^{\mathrm{Hv}}}\right]^{\beta}\right\}, a \in A .
\end{gathered}
$$
follows:

The assignment model of HV traffic flow is listed as 


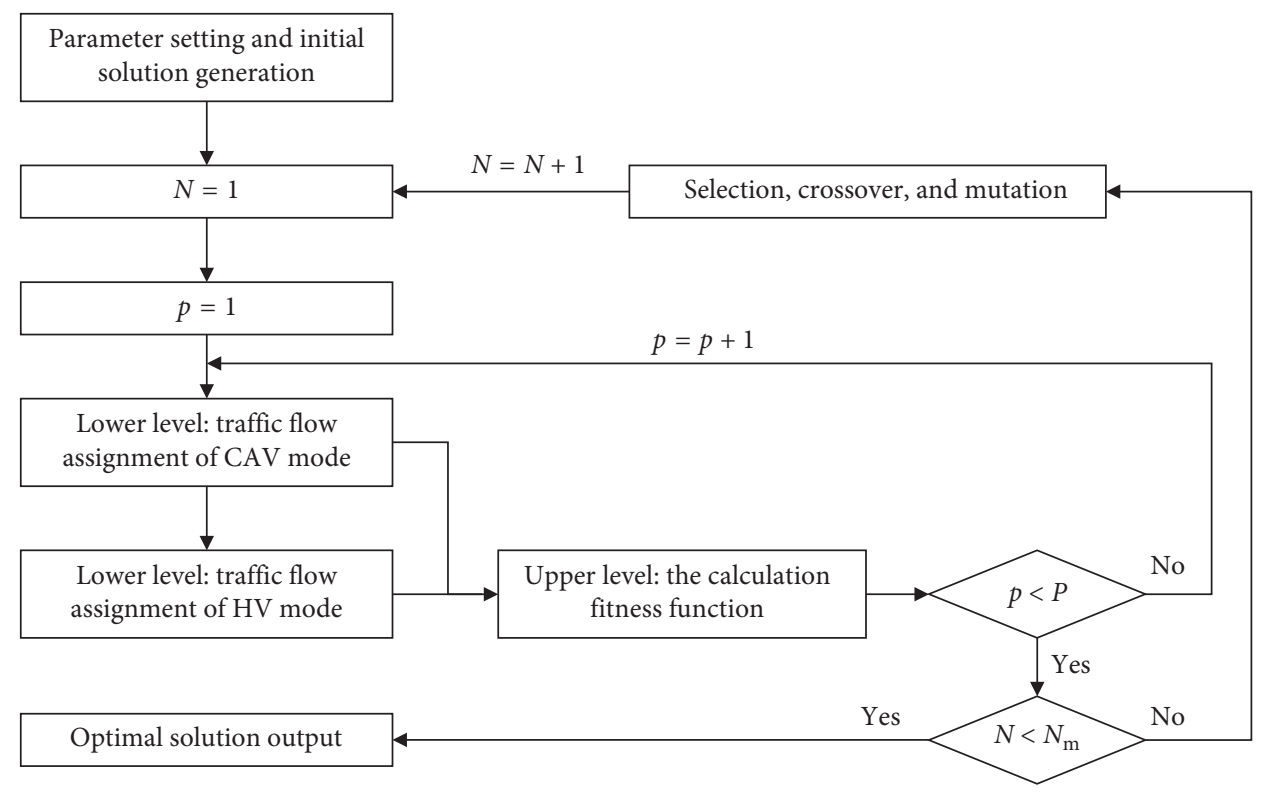

Figure 1: Flow chart of algorithm.

$$
\begin{gathered}
\min \sum_{a \in A} \int_{0}^{x_{a}^{\mathrm{Hv}}} t_{a}^{\mathrm{Hv}}\left(x_{a}^{\mathrm{Cv}}, x_{a}^{\mathrm{Hv}}, n_{a}^{\mathrm{Cv}}\right) \mathrm{d} \omega, \\
\text { s.t. } \quad \sum_{k \in P_{\mathrm{rs}}} f_{\mathrm{rs}}^{\mathrm{Hk}}=q_{\mathrm{rs}}^{\mathrm{Hv}}, \quad(r, s) \in \mathrm{RS}, \\
f_{\mathrm{rs}}^{\mathrm{Hk}} \geq 0, k \in P_{\mathrm{rs}}, \quad(r, s) \in \mathrm{RS}, \\
x_{a}^{\mathrm{Hv}}=\sum_{(r, s) \in \mathrm{RS}} \sum_{k \in P_{\mathrm{rs}}} f_{\mathrm{rs}}^{\mathrm{Hk}} \delta_{\mathrm{rs}}^{\mathrm{Hk}}, \quad a \in A, \\
t_{a}^{\mathrm{Hv}}=t_{a}^{0}\left\{1+\alpha\left[\frac{x_{a}^{\mathrm{Hv}}+\left(1-\eta_{a}\right) x_{a}^{\mathrm{Cv}}}{\left(n_{a}-n_{a}^{\mathrm{Cv}}\right) C_{l}^{\mathrm{Hv}}}\right], \quad a \in A,\right.
\end{gathered}
$$

where equations (7) and (12) are flow conservation constraints; equations (8) and (13) are nonnegative constraints on the route flow; equations (9) and (14) describe the relationship between the link flow and route flow; and equations (10) and (15) are Bureau of Public Road (BPR) functions which are undetermined coefficients.

\section{Solution Algorithm}

In view of the complexity of the bilevel programming model, this paper creatively utilizes the genetic algorithm to search the optimal scheme. The specific steps of the algorithm are listed and shown in Figure 1.

Step 1 (initialization): relevant parameters of the genetic algorithm are defined, including population size $P$, generation gap, crossover probability, mutation probability, and maximum evolution number $N_{m}$. This algorithm uses integer coding: the specific form of coding is $\left\{n_{1}^{\mathrm{Cv}}, n_{1}^{\mathrm{Cv}}, \ldots, n_{a}^{\mathrm{Cv}}\right\}$, whose values range among $\left\{0,1, \ldots,\left(n_{a}-1\right)\right\}$. Let generation $N=1$ and

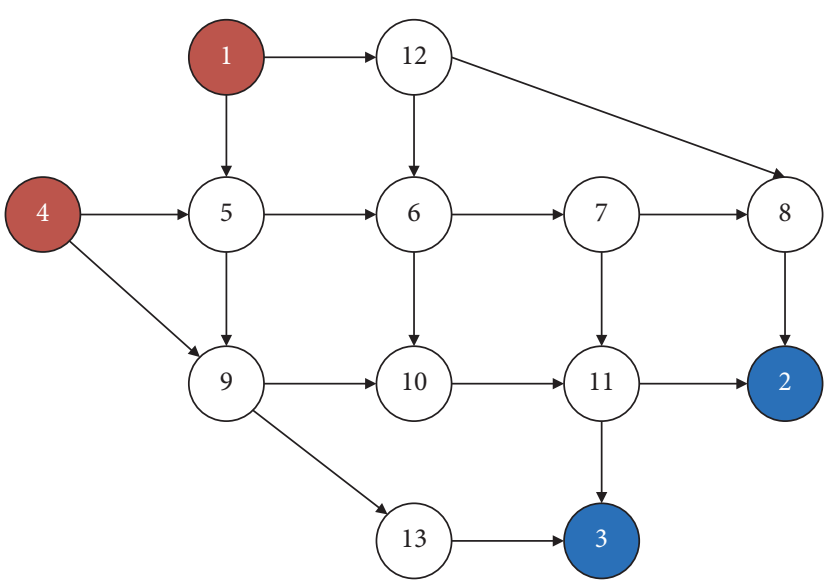

FIGURE 2: Nguyen-Dupuis network schematic diagram.

TABle 1: Basic traffic demand of each OD pair.

\begin{tabular}{lccc}
\hline OD & Origin & Destination & Demand \\
\hline 1 & 1 & 2 & 600 \\
2 & 1 & 3 & 900 \\
3 & 4 & 2 & 750 \\
4 & 4 & 3 & 750 \\
\hline
\end{tabular}

population $p=1$, and randomly generate the CAVLs deployment scheme.

Step 2 (traffic assignment model of lower level): the Frank-Wolfe (F-W) algorithm [29] is used to solve the traffic flow assignment model of the CAV mode. The link cost is updated according to the equilibrium result, and choose it as the initial cost of the HV mode. After that, our research team continues to solve the traffic flow assignment model of the HV mode with the F-W 
TABle 2: Parameters of the Nguyen-Dupuis network.

\begin{tabular}{|c|c|c|c|c|c|}
\hline Link & Origin & Destination & Number of lanes & Free flow time & Current lane capacity \\
\hline 1 & 1 & 5 & 2 & 4 & 150 \\
\hline 2 & 1 & 12 & 3 & 6 & 150 \\
\hline 3 & 4 & 5 & 2 & 5 & 150 \\
\hline 4 & 4 & 9 & 2 & 8 & 150 \\
\hline 5 & 5 & 6 & 4 & 4 & 150 \\
\hline 6 & 5 & 9 & 3 & 10 & 150 \\
\hline 7 & 6 & 7 & 4 & 4 & 150 \\
\hline 8 & 6 & 10 & 3 & 8 & 150 \\
\hline 9 & 7 & 8 & 4 & 4 & 150 \\
\hline 10 & 7 & 11 & 3 & 10 & 150 \\
\hline 11 & 8 & 2 & 2 & 6 & 150 \\
\hline 12 & 9 & 10 & 4 & 4 & 150 \\
\hline 13 & 9 & 13 & 2 & 8 & 150 \\
\hline 14 & 10 & 11 & 4 & 4 & 150 \\
\hline 15 & 11 & 2 & 3 & 5 & 150 \\
\hline 16 & 11 & 3 & 2 & 7 & 150 \\
\hline 17 & 12 & 6 & 2 & 4 & 150 \\
\hline 18 & 12 & 8 & 2 & 12 & 150 \\
\hline 19 & 13 & 3 & 2 & 6 & 150 \\
\hline
\end{tabular}

TABle 3: Optimal scheme of CAVLs.

\begin{tabular}{lccccccccccccccccccccc}
\hline Link & 1 & 2 & 3 & 4 & 5 & 6 & 7 & 8 & 9 & 10 & 11 & 12 & 13 & 14 & 15 & 16 & 17 & 18 & 19 & 11 \\
Number of CAVLs & 0 & 0 & 0 & 0 & 0 & 1 & 0 & 0 & 1 & 0 & 0 & 1 & 0 & 0 & 0 & 0 & 0 & 0 & 0 & 0 \\
\hline
\end{tabular}

algorithm, and the results of the two assignment results are transmitted back to the upper level.

Step 3 (calculate the fitness function of upper level): The objective function of bilevel programming established in this paper is the system cost with the value greater than zero, and this is a minimization problem. Hence, the reciprocal of the objective function is chosen as the fitness function, and the fitness of each individual is calculated according to $x_{a}^{\mathrm{Cv}}, t_{a}^{\mathrm{Cv}}, x_{a}^{\mathrm{Hv}}$, and $t_{a}^{\mathrm{Hv}}$ which can be solved from the lower level.

Step 4: let $p=p+1$. Repeat Steps 2 and 3 until $p \geq P$. Step 5 (iteration): evolutionary operations such as selection, crossover, and mutation [30] are carried out according to the fitness of individuals, and then the population is updated.

Step 6: let $N=N+1$. Repeat Steps 2 to 5 until $N=N_{m}$; then, we get the optimal solution.

\section{Numerical Examples}

4.1. Basic Settings and Results. This paper uses the Nguyen and Dupuis test network as a case study. The network has 13 nodes, 19 links, and 4 OD pairs [31]. The basic topology of the network is shown in Figure 2, where the red node represents the traffic demand generation point and the blue node represents the traffic demand attraction point. Table 1 shows the OD traffic demand. Table 2 is the basic attribute information of the network including the number of lanes, free-flow time, and current lane capacity.

According to the existing literature [25], the basic traffic capacity of a CAV lane is about twice of an HV lane, and

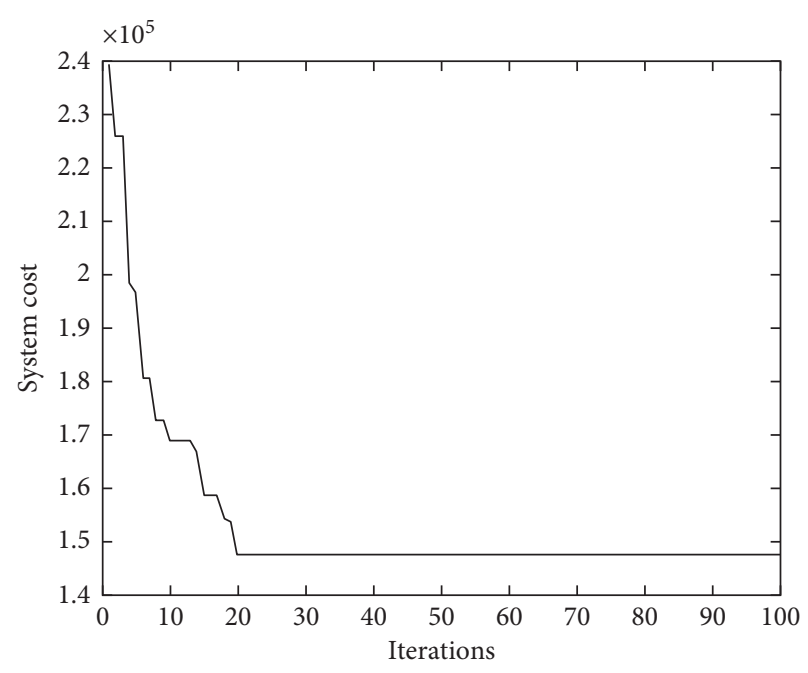

FIGURE 3: Evolutionary process diagram of algorithm.

TABLE 4: Lane deployment schemes for different MPR.

\begin{tabular}{lcccccc}
\hline MPR (\%) & Link & Link & Link & Link & Link & \multirow{2}{*}{ Other links } \\
\hline 10 & 6 & 9 & 12 & 14 & 18 & 0 \\
20 & 1 & 0 & 2 & 0 & 0 & 0 \\
30 & 1 & 2 & 0 & 0 & 0 & 0 \\
40 & 1 & 1 & 1 & 0 & 0 & 0 \\
50 & 1 & 1 & 1 & 1 & 0 & 0 \\
60 & 0 & 2 & 1 & 2 & 0 & 0 \\
70 & 2 & 0 & 2 & 0 & 1 & 0 \\
80 & 1 & 1 & 3 & 0 & 0 & 0 \\
90 & 2 & 2 & 2 & 0 & 0 & 0 \\
\hline
\end{tabular}




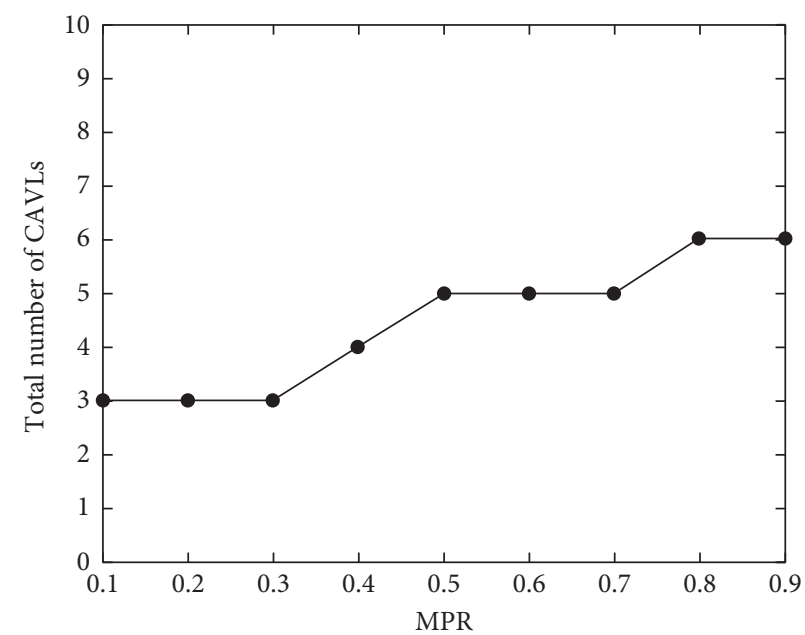

(a)

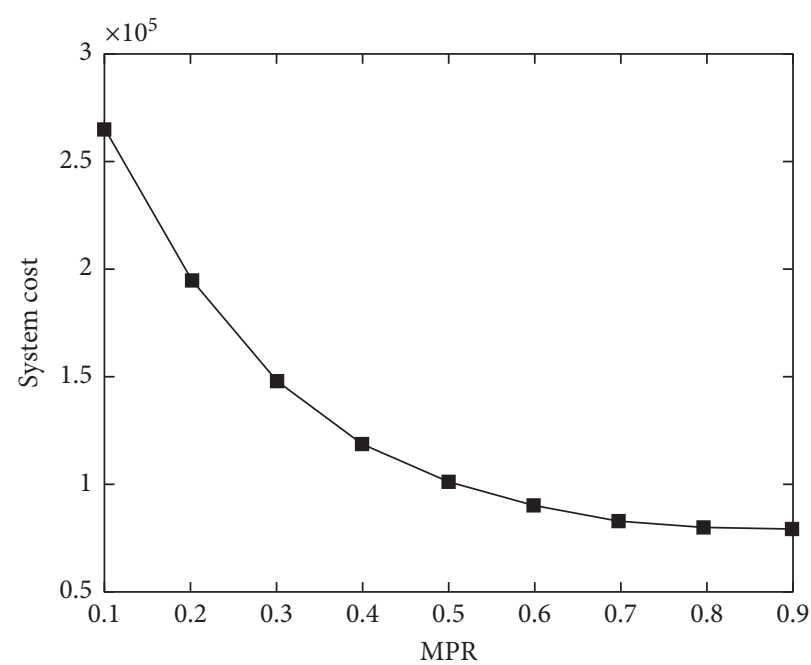

(b)

FIgURE 4: Sensitivity analysis of the CAV MPR. (a) The total number of CAVLs. (b) System cost.

CACC strategy is effective only when CACC MPR is more than $30 \%$. The management cost of CAVLs includes construction cost and maintenance cost with the management cost of a single CAVL is 500 . Let $\alpha$ be 0.15 and $\beta$ be 4.0 in the BPR function. The basic parameters of the genetic algorithm, respectively, are the population size is 50 , generation gap is 0.9 , crossover probability is 0.75 , mutation probability is 0.05 , and maximum evolution times is 100 . When CAV MPR reaches 30\%, the lane deployment scheme is shown in Table 3 , and the running process of the algorithm is shown in Figure 3. As can be seen from Figure 3, the algorithm converges when iterations reach the 20th generation. The total number of CAVLs is 3 with the management cost is 1500. In addition, the system cost is 147416.8 and the system travel cost is 145916.8 .

4.2. Sensitivity Analysis. In this section, the authors mainly analyze the impact of market penetration rate of CAVs, traffic demands, and the capacity of the CAVLs in the proposed method.

4.2.1. Market Penetration Rate of CAVs. In the future, the market will inevitably experience a long transition phase of CAVs coexisting with HVs as connected and automated driving technology. Therefore, it is greatly necessary to analyze the influence of this method when CAV MPR changes.

Fixing the traffic demand of each OD pair and the capacity of the CAVLs, the CAV MPR is adjusted between $10 \%$ and $90 \%$. Then, the genetic algorithm designed in this paper was used to solve the bilevel programming model. Considering the local convergence of the genetic algorithm, ten experiments were carried out on each group of parameters, and the minimum target was chosen as the final result, which is shown in Table 4 and Figure 4.
As can be seen from Table 4 and Figure 4(a), the total number of CAVLs can be the same with the increase of CAV MPR, but the deployment location of the lanes is different. This analysis shows that MPR in the planning year needs to be accurately estimated when lanes are set up, and it also shows the necessity of CAVLs deployment considering from the network level. The traffic volume of CAV increases with the increase of MPR. Only when the traffic volume of CAV reaches a certain scale and the cost of adding CAVLs is lower than the increase of the travel cost, the strategy of adding CAVLs is beneficial. According to the statistics of CAV lanes under all the MPRs, it is found that the probability of CAV lanes set in links $6,9,12$, and 14 is the greatest, which also verifies the feasibility of the calculation results shown in this paper.

Likewise, the system cost shows a downward trend with the increasing of CAV MPR, which declines rapidly in the early stage and slowly in the later stage as seen in Figure 4(b). This is because the MPR is gradually approaching its critical value with the increasing of CAV MPR accompanied with the utilization rate of CAVLs and the actual capacity of the link increasing. However, when the CAV MPR exceeds its critical value, the utilization rate of HV lanes decreases together with the actual capacity of the link. In the latter stage, the increase of CAV MPR has little effect on the decreasing of the travel time due to the traffic demand constraints. If the number of CAVLs continue to increase, it will add to the cost of lane management together with increasing the system cost of the system at the same time.

4.2.2. Traffic Demand. During the design of CAVLs, the impact of traffic demand on the decision-making scheme is also needed to be analyzed except considering the change of CAV MPR. In addition, the intrinsic mechanism of the model is complex with many factors involved in traffic demand forecasting. Additionally, it is necessary to 


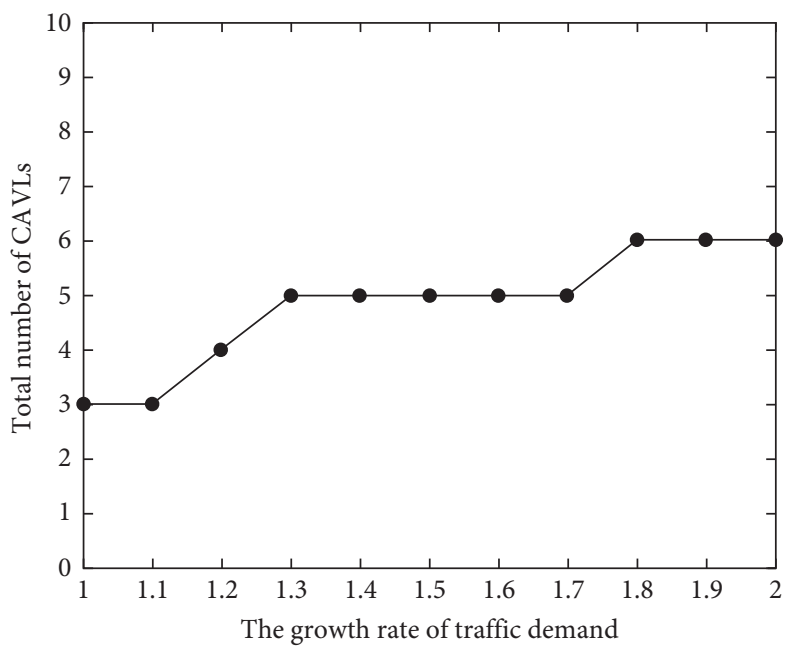

(a)

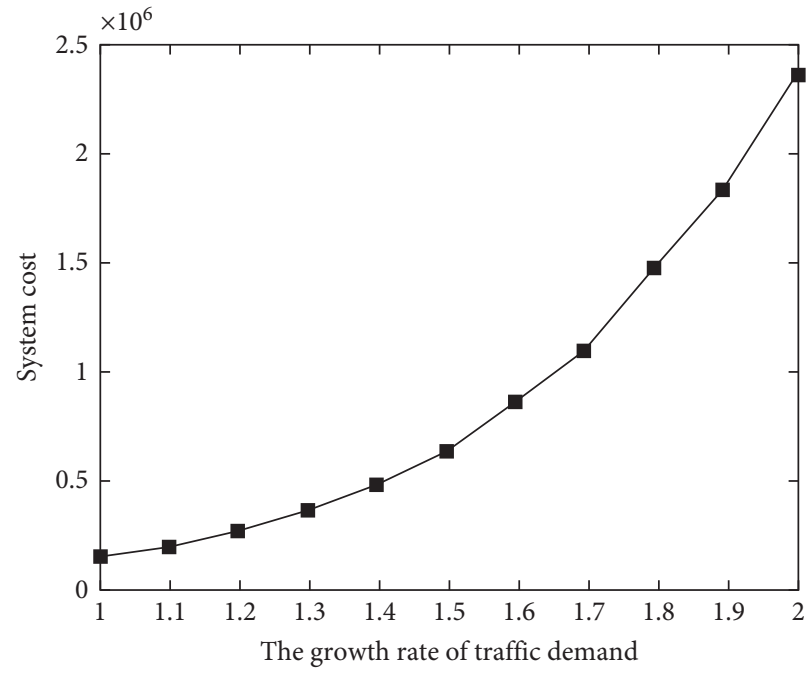

(b)

FIGURE 5: Sensitivity analysis of traffic demand. (a) The total number of CAVLs. (b) System cost.

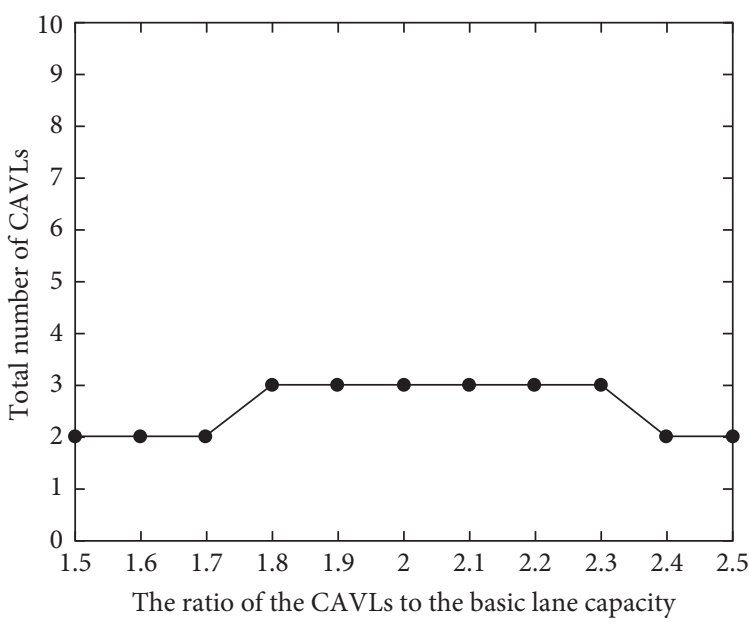

(a)

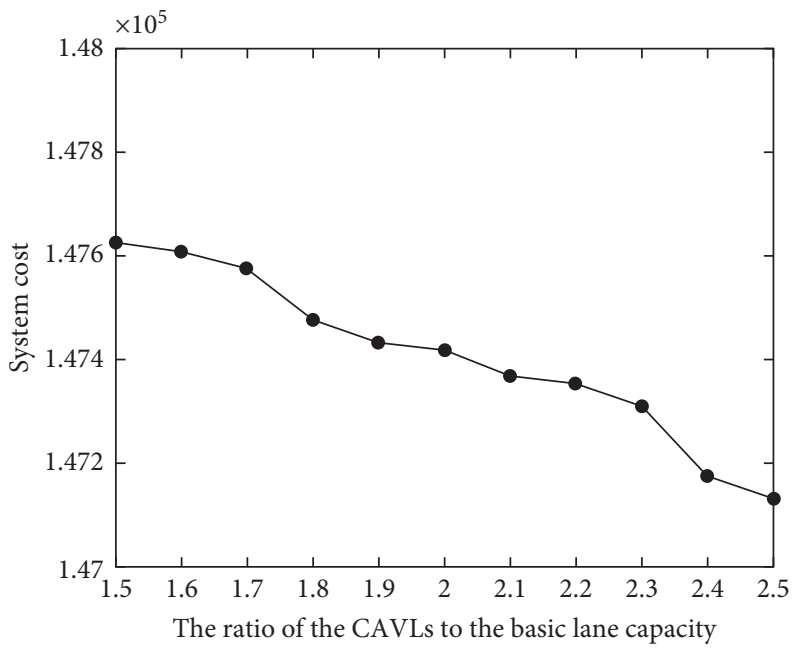

(b)

Figure 6: Sensitivity analysis of the capacity of CAVLs. (a) The total number of CAVLs. (b) System cost.

consider the variations of system performance under different traffic demands with many uncertainties in the actual situation. Therefore, CAV MPR is fixed and located at $30 \%$. The total traffic demand is multiplied by the growth factor of $100 \%-200 \%$ compared with the original basis, while the proportion of OD traffic demand remains unchanged.

In addition, the total number of CAVLs is the same (Figure 5(a)) when the growth rate of traffic demand varies between 1.3 and 1.7, which results from the optimal utilization of lane function by the route selection behavior of CAVs. The total number of CAVLs also doubles when traffic demand doubles. In addition, Figure 5(b) shows that the system cost increases exponentially with the increase of traffic demand.
4.2.3. Capacity of the CAVLs. Since the capacity of the CAVLs is highly sensitive to the performance of CAV in terms of its average headway, sensitivity analysis on the capacity of the CAV lane would be necessary. Therefore, fixing the CAV MPR and the traffic demand, the capacity of CAVLs is multiplied by the factor of $150 \%-300 \%$ compared with the basic lane capacity. The results of the total number of CAVLs and the system cost are shown in Figure 6(a) and Figure 6(b), respectively.

As can be seen from Figure 6(a), the total number of CAVLs shows a trend of increasing first and decreasing later, and its values are the same when the ratio of CAVLs to the basic lane capacity varies between 1.8 and 2.3. When the capacity of CAVLs is low, the cost of adding CAVLs is greater than the reduction of system travel cost, so CAVLs 
are deployed in a small number. When the capacity of CAVLs is high, fewer CAVLs can meet the traffic demand. In addition, Figure 6(b) shows that the system cost decreases with the increase of CAVLs capacity.

\section{Conclusions}

To sum up, this paper proposes an optimization method for CAVL deployment plan, which aims to solve the problem with capacity drops when HVs are mixed into CACC systems. This method creatively simplified the CAVL deployment into a traffic network design problem and established a bilevel primary-secondary programming model. The upper level generated the decision-making scheme of the lane deployment scheme, and the lower level assigned the traffic flow including the CAV mode and HV mode according to the decision-making scheme of the upper level. The equilibrium results of the lower level were used to evaluate the performance of the upper level. Due to the characteristics of the model, a genetic algorithm was designed to solve this model. The numerical results show that the proposed method can obtain a feasible scheme with the consideration of both the system cost and the management cost for CAVLs. The sensitivity analysis results of CAV MPR, traffic demands, and the capacity of CAVLs further validate the feasibility and flexibility of the proposed method.

However, there are also some limitations where HVs give preferences to CAVs when assigning flow in the lower-level model, which is different from the general situation with two-mode flow assigned simultaneously. Regarding to the value of lane management cost, it needs to be quantified more accurately by considering from much more aspects. Due to the large number of variables when applying the proposed model in large-size network, the genetic algorithm may not converge. A combination algorithm combining the advantages of genetic algorithm and active set algorithm will be proposed in future studies.

\section{Abbreviations}

$G(V, A)$ : Traffic network, where $V$ is the node of the traffic network and $A$ is the link set

$D: \quad$ Set of links with CAVLs

$\bar{D}: \quad$ Set of links without CAVLs

RS: $\quad$ Set of demand of OD pair

$x_{a}$ : $\quad$ Flow of link $a$

$x_{a}^{\mathrm{Hv}}$ : $\quad$ Flow of HVs on link $a$

$x_{a}^{\mathrm{Cv}}$ : $\quad$ Flow of CAVs on link $a$

$f_{\mathrm{rs}}^{\mathrm{Cv}, k}$ : Route flow of CAVs

$f_{\mathrm{rs}}^{\mathrm{Hv}, k}: \quad$ Route flow of HVs

$t_{a}^{0}: \quad$ Link cost of link a under free flow

$t_{a}(\bullet)$ : Cost function of link $a$

$t_{a}^{\mathrm{Cv}}$ : $\quad$ Cost function of link $a \in D$

$t_{a}^{\mathrm{Hv}}: \quad$ Cost function of link $a \in \bar{D}$

$w_{C}$ : Management cost of single CAVL

$\eta_{a}: \quad 0-1$ variables; if $a \in D$, then $\eta_{a}=1$; otherwise, $\eta_{a}=0$

$t_{a}^{0}$ : Link cost of link a under free flow
$C_{l}^{\mathrm{Hv}}$ : $\quad$ Basic capacity of HV lane

$C_{l}^{\mathrm{Cv}}$ : $\quad$ Basic capacity of CAVLs

$n_{a}$ : Total number of lanes of link $a$

$n_{a}^{\mathrm{Hv}}: \quad \quad$ Number of HV lanes on link $a$

$n_{a}^{\mathrm{Cv}}: \quad$ Number of CAVLs on link $a$.

\section{Data Availability}

The data used to support the findings of this study are available from the corresponding author upon request.

\section{Conflicts of Interest}

The authors declare that there are no conflicts of interest regarding the publication of this paper.

\section{Acknowledgments}

This study was supported by the National Natural Science Foundation of China under Grant nos. 61773288, 51808057, and 51678076 . This study was in part supported by National Key Research and Development Program of China (no. 2018YFB1600805).

\section{References}

[1] T. X. Li and K. M. Kockelman, "Valuing the safety benefits of connected and automated vehicle technologies," in Proceedings of the 95st Transportation Research Board Annual Meeting, San Francisco, CA, USA, September 2016.

[2] J. Liu and A. J. Khattak, "Delivering improved alerts, warnings, and control assistance using basic safety messages transmitted between connected vehicles," Transportation Research Part C: Emerging Technologies, vol. 68, pp. 83-100, 2016.

[3] C. Letter and L. Elefteriadou, "Efficient control of fully automated connected vehicles at freeway merge segments," Transportation Research Part C: Emerging Technologies, vol. 80, pp. 190-205, 2017.

[4] P. Tientrakool, Y. C. Ho, and N. F. Maxemchuk, "Highway capacity benefits from using vehicle-to-vehicle communication and sensors for collision avoidance," in Proceedings of the 2011 IEEE Vehicular Technology Conference (VTC Fall), San Francisco, CA, USA, September 2011.

[5] H. Jiang, J. Hu, S. An, M. Wang, and B. B. Park, "Eco approaching at an isolated signalized intersection under partially connected and automated vehicles environment," Transportation Research Part C: Emerging Technologies, vol. 79, pp. 290-307, 2017.

[6] H. Yang, H. Rakha, and M. V. Ala, "Eco-cooperative adaptive cruise control at signalized intersections considering queue effects," IEEE Transactions on Intelligent Transportation Systems, vol. 18, no. 6, pp. 1575-1585, 2011.

[7] Z. Wang, G. Y. Wu, and M. J. Barth, "Cooperative eco-driving at signalized intersections in a partially connected and automated vehicle environment," IEEE Transactions on Intelligent Transportation Systems, vol. 21, pp. 1-10, 2019.

[8] A. Talebpou and H. S. Mahmassani, "Influence of connected and autonomous vehicles on traffic flow stability and throughput," Transportation Research Part C: Emerging Technologies, vol. 71, pp. 143-163, 2016. 
[9] F.-Y. Wang, "Parallel control and management for intelligent transportation systems: concepts, architectures, and applications," IEEE Transactions on Intelligent Transportation Systems, vol. 11, no. 3, pp. 630-638, 2010.

[10] L. Li, W.-L. Huang, Y. Liu, N.-N. Zheng, and F.-Y. Wang, "Intelligence testing for autonomous vehicles: a new approach," IEEE Transactions On Intelligent Vehicles, vol. 1, no. 2, pp. 158-166, 2016.

[11] G. Marsden, M. McDonald, and M. Brackstone, "Towards an understanding of adaptive cruise control," Transportation Research Part C: Emerging Technologies, vol. 9, no. 1, pp. 33-51, 2001.

[12] L. Xiao and F. Gao, "A comprehensive review of the development of adaptive cruise control systems," Vehicle System Dynamics, vol. 48, no. 10, pp. 1167-1192, 2010.

[13] L. Li and X. Li, "Parsimonious trajectory design of connected automated traffic," Transportation Research Part B: Methodological, vol. 119, pp. 1-21, 2019.

[14] L. Xiao, M. Wang, W. Schakel, and B. van Arem, "Unravelling effects of cooperative adaptive cruise control deactivation on traffic flow characteristics at merging bottlenecks," Transportation Research Part C: Emerging Technologies, vol. 96, pp. 380-397, 2018.

[15] F. Chen and S. Chen, "Injury severities of truck drivers in single- and multi-vehicle accidents on rural highways," Accident Analysis \& Prevention, vol. 43, no. 5, pp. 1677-1688, 2011.

[16] F. Chen, H. R. Peng, X. X. Ma, J. Y. Liang, W. Hao, and X. D. Pan, "Examining the safety of trucks under crosswind at bridge-tunnel section: a driving simulator study," Tunnelling and Underground Space Technology, vol. 92, p. 103034, 2019.

[17] Q. Zeng, W. Gu, X. Zhang, H. Wen, J. Lee, and W. Hao, "Analyzing freeway crash severity using a Bayesian spatial generalized ordered logit model with conditional autoregressive priors," Accident Analysis \& Prevention, vol. 127, pp. 87-95, 2019.

[18] Q. Zeng, H. Wen, H. Huang, and M. Abdel-Aty, “A Bayesian spatial random parameters Tobit model for analyzing crash rates on roadway segments," Accident Analysis \& Prevention, vol. 100 , pp. 37-43, 2017.

[19] Y. Y. Qin, H. Wang, W. Wang, and Q. Wan, "Fundamental diagram model of heterogeneous traffic flow mixed with cooperative adaptive cruise control vehicles and adaptive cruise control vehicles," China Journal of Highway and Transport, vol. 30, no. 10, pp. 127-136, 2017.

[20] L. Ye and T. Yamamoto, "Impact of dedicated lanes for connected and autonomous vehicle on traffic flow throughput," Physica A: Statistical Mechanics and Its Applications, vol. 512, pp. 588-597, 2018.

[21] A. Ghiasi, O. Hussain, Z. Qian, and X. Li, "A mixed traffic capacity analysis and lane management model for connected automated vehicles: a Markov chain method," Transportation Research Part B: Methodological, vol. 106, pp. 266-292, 2017.

[22] Z. Chen, F. He, L. Zhang, and Y. Yin, "Optimal deployment of autonomous vehicle lanes with endogenous market penetration," Transportation Research Part C: Emerging Technologies, vol. 72, pp. 143-156, 2016.

[23] D. Chen, S. Ahn, M. Chitturi, and D. A. Noyce, "Towards vehicle automation: roadway capacity formulation for traffic mixed with regular and automated vehicles," Transportation Research Part B: Methodological, vol. 100, pp. 196-221, 2017.

[24] H. Liu, X. Kan, S. E. Shladover, X.-Y. Lu, and R. E. Ferlis, "Modeling impacts of cooperative adaptive cruise control on mixed traffic flow in multi-lane freeway facilities,"
Transportation Research Part C: Emerging Technologies, vol. 95, pp. 261-279, 2018.

[25] A. Talebpour, H. S. Mahmassani, and A. Elfar, "Investigating the effects of reserved lanes for autonomous vehicles on congestion and travel time reliability," Transportation Research Record: Journal of the Transportation Research Board, vol. 2622, no. 1, pp. 1-12, 2017.

[26] H. Yang and M. G. H. Bell, "A capacity paradox in network design and how to avoid it," Transportation Research Part A: Policy and Practice, vol. 32, no. 7, pp. 539-545, 1998.

[27] D. Braess, A. Nagurney, and T. Wakolbinger, "On a paradox of traffic planning," Transportation Science, vol. 39, no. 4, pp. 446-450, 2005.

[28] Z. Chen, F. He, Y. Yin, and Y. Du, "Optimal design of autonomous vehicle zones in transportation networks," Transportation Research Part B: Methodological, vol. 99, pp. 44-61, 2017.

[29] Y. B. Wang, M. Papageorgiou, and G. Sarros, "Real-time route guidance for large-scale express ring-roads," in Proceedings of IEEE Intelligent Transportation Systems Conference, Toronto, Ont., Canada, September 2007.

[30] F. Shi, H. Y. Su, and X. Wang, "Design of reversible lanes with tidal flow on road network," Journal of Transportation Systems Engineering and Information Technology, vol. 15, no. 4, pp. 57-62, 2015.

[31] S. Nguyen and C. Dupuis, “An efficient method for computing traffic equilibria in networks with asymmetric transportation costs," Transportation Science, vol. 18, no. 2, pp. 185-202, 1984. 